\title{
SOME SAMPLE FUNCTION PROPERTIES OF A PROCESS WITH STATIONARY INDEPENDENT INCREMENTS
}

\author{
BY \\ SHASHANKA SHEKHAR MITRA(1)
}

1. Introduction. Let $X=\{X(t): t \geqq 0\}$ be a stochastic process with stationary independent increments in $R_{1}$. We are mainly interested in studying the convergence properties as $t \rightarrow \infty$ of the sample functions of a process of the type $Y_{a}=\left\{t^{-a} X(t): t \geqq 0\right\}$, where $a$ is a fixed non-negative real number.

A. Khintchine [4] studied the conditions under which $t^{-a} X(t) \rightarrow{ }^{\text {a.s. }} 0$ as $t \rightarrow \infty$, subject to the restriction that $X$ is a stable process of index $\alpha$ where $0<\alpha \leqq 2$. In this case he has shown that if $a>1 / \alpha$, then $t^{-a} X(t) \rightarrow^{\text {a.s. }} 0$.

It should be remarked that the problem of the behavior of the sample functions as $t \rightarrow 0$, has already been explored by R. M. Blumenthal and R. K. Getoor [1] and by $\mathrm{A}$. Khintchine [5].

In the present paper we obtain results pertaining to convergence to zero, without the restriction that $X$ be a stable process. Our result is that with regard to any process of the type under investigation, there is associated a lower bound $1 / \theta$, such that for any $a>1 / \theta, t^{-a} X(t) \rightarrow^{\text {a.s. }} 0$ as $t \rightarrow \infty$. We also prove that under certain restrictive conditions, $t^{-a}|X(t)| \rightarrow^{\text {a.s. }} \infty$, provided $a$ is chosen sufficiently small. It is noteworthy that a stable process of index $\alpha$ with $0<\alpha<1$, always satisfies this condition and for such a case $t^{-a}|X(t)| \rightarrow^{\text {a.s. }} \infty$, provided $a<(1-\alpha) / \alpha$. One of the results of the work is that given any $a>0$, it is not always true that $t^{-a}|X(t)|$ will either converge to zero or diverge to infinity with probability one. There are cases such that $\limsup _{t \rightarrow \infty} t^{-a}|X(t)|=\infty$ a.s. and $\liminf _{t \rightarrow \infty} t^{-a}|X(t)|=0$ a.s. for some suitably chosen $a$. Thus in particular if $X$ is a symmetric stable process with index $\alpha>1$, then for every $a<1 / \alpha$, $\lim \sup _{t \rightarrow \infty} t^{-a}|X(t)|=\infty$ a.s. and $\liminf _{t \rightarrow \infty} t^{-a}|X(t)|=0$ a.s.

2. Preliminaries. Let $X=\{X(t): t \geqq 0\}$ be a stochastic process in $R_{1}$ with stationary independent increments. It is assumed that the process is defined over some basic probability space $(\Omega, \mathscr{F}, P)$. For the sake of brevity, the $\omega$ 's will very often be deleted and we shall write our process the way it is written in the first line of this section. It is assumed further that $X(0)=0 \mathrm{a} . \mathrm{s}$. and that almost all sample functions are right continuous. If $\phi_{t-s}$ is the characteristic function of $X(t)-X(s)$ with $t>s$, then $\phi_{t-s}(y)=\exp [-(t-s) \Psi(y)]$, where

Received by the editors September 15, 1961 and, in revised form, June 21, 1962.

(1) Currently at the University of Arizona, Tucson, Arizona. 


$$
\Psi(y)=i a y+\sigma^{2} y^{2}+\int_{-\infty}^{\infty}\left(1-e^{i x y}+\frac{i x y}{1+x^{2}}\right) v(d x),
$$

with $a$ and $\sigma$ any two real numbers and $v$ a (Borel) measure on $R_{1}$ satisfying

$$
\int_{-\infty}^{\infty} \frac{x^{2}}{1+x^{2}} v(d x)<\infty, \quad v\{0\}=0 .
$$

The quantities $a, \sigma^{2}$ and $v$ appearing in (2.1) are uniquely determined by $\phi$. The function $\Psi$ is called the exponent of $X$ and $v$ is called the Lévy measure of $X$. Throughout this paper we shall use the symbols $X_{t}(\omega)$ and $X(t, \omega)$ interchangeably. Under the assumptions made toward the beginning of this section one can easily verifiy the following two properties:

P1: $X(t, \omega)$ is a $T \times \Omega$ measurable function with respect to the Borel field $\mathscr{B}(T) \times \mathscr{F}$ where $\mathscr{B}(T)$ is the Borel field of Borel sets on the positive half line and the product Borel field is obtained according to the conventional way.

P2: Given an $\varepsilon>0$, a $\delta$ such that $0<\delta<1$ and a $T>0$, such that the condition

$$
P\{|X(t)| \leqq \varepsilon\}>1-\delta
$$

hold for all $t \leqq T$, then for any $s \geqq 0$,

$$
P[\underset{s \leqq k \leqq s+T}{\text { L.U.B. }}|X(k)-X(s)|>2 \varepsilon] \leqq \frac{\delta}{1-\delta} .
$$

We want to introduce in the following section a set of definitions and notations to be used later on.

\section{Notation and definitions.}

3.1. $\gamma=\sup \left[\alpha: \quad \int_{|x| \geqq 1}|x|^{\alpha} v(d x)<\infty\right]$,

3.2. $\theta=\sup \left[\alpha:|y|^{-\alpha} \operatorname{Re} \psi(y) \rightarrow 0\right.$ as $\left.y \rightarrow 0\right]$,

3.3. $\theta^{\prime}=\inf \left[\alpha:|y|^{-\alpha} \operatorname{Re} \psi(y) \rightarrow \infty\right.$ as $\left.y \rightarrow 0\right]$,

3.4. $\eta=\sup \left[\alpha:|y|^{-\alpha}|\operatorname{Im} \psi(y)| \rightarrow 0\right.$ as $\left.y \rightarrow 0\right]$,

3.5. $\eta^{\prime}=\inf \left[\alpha:|y|^{-\alpha}|\operatorname{Im} \psi(y)| \rightarrow \infty\right.$ as $\left.y \rightarrow 0\right]$,

3.6. $\delta=\sup \left[\alpha: y^{-\alpha} \int_{0}^{\infty}\left(1-e^{-r y}\right) v(d r) \rightarrow 0\right.$ as $\left.y \downarrow 0\right]$,

3.7. $\delta^{\prime}=\inf \left[\alpha: y^{-\alpha} \int_{0}^{\infty}\left(1-e^{-r y}\right) v(d r) \rightarrow \infty\right.$ as $\left.y \downarrow 0\right]$.

In 3.6 and 3.7 we assume that $\int_{0}^{1} r v(d r)<\infty$.

4. Lévy measure and the characteristic function. In this section we propose to find certain relationships between the various indices described in the previous section. We shall assume throughout the paper that $\operatorname{Re} \psi(y) \not \equiv 0$.

LEMMA 4.1. The indices $\theta, \theta^{\prime}, \gamma$ and $\eta$ satisfy:
(a) $\theta \geqq \min (2, \gamma)$,
(b) $\eta \geqq \min (1, \gamma)$,
(c) $\theta^{\prime} \leqq 2$. 
Proof. In order to prove (a) we first note that $1-\cos t \leqq 2|t|^{\alpha}$ for all $\alpha$ such that $0 \leqq \alpha<2$. Next take any arbitrary $\gamma_{1}$ such that $\gamma_{1}<\min (2, \gamma)$. Writing

$$
\begin{aligned}
|y|^{-\gamma_{1}} \operatorname{Re} \psi(y) & =|y|^{-\gamma_{1}} \sigma^{2} y^{2}+|y|^{-\gamma_{1}} \int_{|x| \leqq 1}(1-\cos x y) v(d x) \\
& +\int_{|x|>1}(1-\cos x y) v(d x) \\
& \leqq|y|^{-\gamma_{1}} \sigma^{2} y^{2}+2|y|^{-\gamma_{1}} y^{2} \int_{|x| \leqq 1} x^{2} v(d x) \\
& +2|y|^{-\gamma_{1}}|y|^{\gamma_{2}} \int_{|x|>1}|x|^{\gamma_{2}} v(d x),
\end{aligned}
$$

where $\gamma_{2}$ is some number such that $\gamma_{1}<\gamma_{2}<\min (2, \gamma)$. Proof of (a) now follows from Lebesgue's theorem on dominated convergence.

Turning to the proof of (b) we first note that $|\sin t-t| \leqq 2 t^{2}$ and $|\sin t| \leqq|t|^{\gamma^{\prime \prime}}$ where $\gamma^{\prime \prime}<1$. Next, taking a $\gamma^{\prime}$ such that $\gamma^{\prime}<\min (1, \gamma)$, we write

$$
\begin{aligned}
|y|^{-\gamma^{\prime}}|\operatorname{Im} \psi(y)|<|y|^{-\gamma^{\prime}} a|y|+|y|^{-\gamma^{\prime}} & \int_{-\infty}^{\infty} \frac{|x y-\sin x y|}{1+x^{2}} v(d x) \\
& +|y|^{-\gamma^{\prime}} \int_{-\infty}^{\infty} \frac{x^{2}|\sin x y|}{1+x^{2}} v(d x) .
\end{aligned}
$$

The proof follows from the fact that

$$
\int_{-\infty}^{\infty} \frac{x^{2}|\sin x y|}{1+x^{2}} v(d x) \leqq \int_{-\infty}^{\infty} \frac{x^{2}|x|^{\gamma^{\prime \prime}}|y|^{\gamma^{\prime \prime}}}{1+x^{2}} v(d x)
$$

where $\gamma^{\prime \prime}$ is such that $\gamma^{\prime}<\gamma^{\prime \prime}<\min (1, \gamma)$. The proof of (c) is obvious if $\sigma^{2} \neq 0$. If $\sigma^{2}=0$, then since $\operatorname{Re} \psi(y) \neq \equiv 0$, we have $v \neq 0$, and the conclusion follows from Fatou's lemma.

THEOREM 4.1. The indices $\theta$ and $\gamma$ satisfy the relation $\theta=\min (2, \gamma)$.

Proof. Obviously $\theta \leqq \theta^{\prime}$. Also by Lemma $4.1 \theta^{\prime} \leqq 2$. Hence $\theta \leqq 2$. It follows from Lemma 4.1 that $\theta \geqq \min (2, \gamma)$. Combining all these facts the theorem becomes obvious if $\gamma \geqq 2$. So, let $\gamma<2$. If possible, let $\gamma<\theta$. We can pick $\alpha, \alpha^{\prime}$ such that $\gamma<\alpha^{\prime}<\alpha<\theta$ and such that $|y|^{-\alpha} \operatorname{Re} \psi(y)<\varepsilon$, provided $|y|<1 / 2^{k}$, for some $k>0$. We shall use the inequality $1-\cos t \geqq \frac{1}{4}(1-\cos 1)|t|^{a}$ where $a$ is a real number satisfying $0 \leqq a<2$, and $t$ is such that $1 \leqq t \leqq 2$. Since $\int_{|x|>1}|x|^{\alpha} v(d x)=\infty$, there exists some $n>k+1$, such that 


$$
\int_{2^{n}<|x| \leqq 2^{n+1}}|x|^{\alpha^{\prime}} v(d x)>4 \varepsilon /(1-\cos 1) 2^{n\left(\alpha-\alpha^{\prime}\right)},
$$

using (4.1) and the above inequality with $a=\alpha^{\prime}$, we obtain

$$
\begin{aligned}
2^{n \alpha} \operatorname{Re} \psi\left(2^{-n}\right) & \geqq 2^{n \alpha} \int_{2^{n}<|x| \leqq 2^{n+1}}\left(1-\cos x 2^{-n}\right) v(d x) \\
& \geqq \frac{1}{4}(1-\cos 1) 2^{n\left(\alpha-\alpha^{\prime}\right)} \int_{2^{n}<|x| \leqq 2^{n+1}}|x|^{\alpha^{\prime}} v(d x)>\varepsilon .
\end{aligned}
$$

This leads to a contradiction.

5. Convergence to zero. Let $Y(t)=t^{-1 / \alpha} X(t)$, where $\alpha>0$. For $t \geqq 0$, define

$$
B(t)=\int_{-\infty}^{\infty} e^{-x^{2}} G(t, d x),
$$

where $G(t, A)$ is the distribution of the random variable $Y(t)$. Observe that the integrand is the characteristic function of a Normal Variate. Denoting by $f(x)$ the corresponding density, we have for $a>0$,

$$
\int_{-\infty}^{\infty}|x|^{a} f(x) d x=M_{a},
$$

a finite real number.

Lemma 5.1. For every $\alpha<\min (\theta, \eta)$, there exists $a c>0$, such that $\operatorname{Sup}_{t \geq 0} t^{c}(1-B(t))<\infty$.

Proof. Let $c$ be any positive number which is smaller than $[\min (\theta, \eta)-\alpha] \alpha^{-1}$. Take an $\alpha^{\prime}$ such that $\alpha<\alpha^{\prime}<\min (\theta, \eta)$ and such that $\alpha^{\prime} / \alpha>1+c$. Observe that $|\Psi(y)||y|^{-\alpha^{\prime}} \leqq \operatorname{Re} \Psi(y)|y|^{-\alpha^{\prime}}+|\operatorname{Im} \Psi(y)||y|^{-\alpha^{\prime}}$. Hence, it follows by the choice of $\alpha^{\prime}$ that $|\Psi(y)||y|^{-\alpha^{\prime}} \rightarrow 0$ as $y \rightarrow 0$. In order to prove the theorem we can restrict ourselves to those values of $t$ which are greater than 1 . Now,

$$
\begin{aligned}
B(t) & =\int_{-\infty}^{\infty} e^{-x^{2}} G(t, d x) \\
& =\int_{-\infty}^{\infty}\left[\int e^{i y x} f(y) d y\right] G(t, d x) \\
& =\int_{-\infty}^{\infty} e^{-t \Psi\left(x / t^{1 / \alpha}\right)} f(x) d x .
\end{aligned}
$$

Hence,

$$
1-B(t)=\int_{-\infty}^{\infty}\left(1-e^{-t \Psi\left(x / t^{1 / \alpha}\right.}\right) f(x) d x .
$$


Substituting $x / t^{1 / \alpha}=y$, we obtain

$$
1-B(t)=\int_{-\infty}^{\infty}\left(1-e^{-t \psi(y)}\right) t^{1 / \alpha} f\left(t^{1 / \alpha} y\right) d y
$$

Let $\delta>0$ be such that $|\psi(y)|<|y|^{\alpha^{\prime}}$, if $|y|<\delta$. Since $\operatorname{Re} t \psi(y) \geqq 0$ and $\left|1-e^{-z}\right| \leqq z$ for all complex $z$ such that $\operatorname{Re} z \geqq 0$, we have

$$
\begin{aligned}
t^{c}(1-B(t)) \leqq & \int_{|y|<\delta} t^{c+1}|y|^{\alpha^{\prime}} t^{1 / \alpha} f\left(t^{1 / \alpha} y\right) d y \\
& +2 \delta^{-c \alpha} \int_{|y| \geqq \delta}\left|t^{1 / \alpha} y\right|^{c \alpha} t^{1 / \alpha} f\left(t^{1 / \alpha} y\right) d y .
\end{aligned}
$$

Since $c+1<\alpha^{\prime} / \alpha$ and $t>1$, we finally obtain $t^{c}(1-B(t)) \leqq M_{\alpha^{\prime}}+2 \delta^{-c \alpha} M_{c \alpha}$, where $M_{\alpha^{\prime}}$ and $M_{c \alpha}$ are defined by (5.1).

THEOREM 5.1. If $\alpha<\min (\theta, \eta)$, then $t^{-1 / \alpha} X(t) \rightarrow^{\text {a.s. }} 0$ as $t \rightarrow \infty$.

Proof. Since $\alpha<\min (\theta, \eta)$ we obtain by Lemma 5.1

$$
\begin{aligned}
t^{-c} K>(1-B(t)) & =\int_{-\infty}^{\infty}\left(1-e^{-x^{2}}\right) G(t, d x) \\
& \geqq \int_{|x| \geqq \eta^{\prime}}\left(1-e^{-x^{2}}\right) G(t, d x) \\
& \geqq\left(1-e^{-\eta^{2}}\right) P\left[t^{-1 / \alpha}|X(t)|>\eta^{\prime}\right] \\
& \text { for every } \eta^{\prime}>0 .
\end{aligned}
$$

Hence,

$$
P\left[t^{-1 / \alpha}|X(t)| \geqq \eta^{\prime}\right]<t^{-c} K_{\eta^{\prime}},
$$

where $K_{\eta^{\prime}}$ is some constant depending upon $\eta^{\prime}$. Let $t$ be an arbitrary point of the interval $\left[2^{n}, 2^{n+1}\right]$. Then

$$
\begin{gathered}
P\left[\left|X\left(2^{n+1}\right)-X(t)\right| \geqq \eta^{\prime} 2^{(n / \alpha-1)}\right] \leqq P\left[\left|X\left(2^{n+1}\right)\right|>\eta^{\prime} 2^{(n / \alpha-2)}\right] \\
+P\left[|X(t)| \geqq \eta^{\prime} 2^{(n / \alpha-2)}\right]<2^{1-n c} C_{\eta^{\prime}}
\end{gathered}
$$

by (5.3). Since $2^{n} \leqq t \leqq 2^{n+1}, l \equiv 2^{n+1}-t<2^{n}$. The above inequality implies

$$
P\left[|X(l)| \leqq \eta^{\prime} 2^{(n / \alpha-1)}\right]<2^{1-n c} C \eta^{\prime}=2^{-n c} C_{\eta^{\prime}}^{\prime}
$$

for all $l$ such that $0 \leqq l \leqq 2^{n}$.

Putting $s=2^{n}, T=2^{n}$ in (2.4), we obtain

$$
P\left[\underset{2^{n} \leqq t \leqq 2^{n+1}}{\text { L.U.B. }}|X(t)|>\eta^{\prime} 2^{n / \alpha}\right] \leqq 2^{-n c} \Gamma_{\eta} C_{\eta^{\prime}}^{\prime}
$$


where $\Gamma_{n}=\left(1-2^{-n c} C^{\prime}{ }_{n^{\prime}}\right)^{-1}$. Note that $\Gamma_{n} \rightarrow 1$ as $n \rightarrow \infty$. Relation (5.5) immediately implies

$$
P\left[\underset{2^{n} \leqq t \leqq 2^{2+1}}{\operatorname{L.U.B}} t^{-1 / \alpha}|X(t)|>\eta^{\prime}\right] \leqq 2^{-n c} \Gamma_{n} C_{\eta^{\prime}}^{\prime}
$$

Set

$$
\chi_{n}=\underset{2^{n} \leqq t \leqq 2^{n+1}}{\text { L.U.B. }} t^{-1 / \alpha}|X(t)| .
$$

Since in (5.6) $\eta^{\prime}>0$ is arbitrary it follows from the Borel-Cantelli Lemma that $\chi_{n} \rightarrow^{\text {a.s. }} 0$ as $n \rightarrow \infty$. The Theorem follows.

Corollary 5.1. If $\alpha<\min (1, \gamma)$, then $t^{-1 / \alpha} X(t) \rightarrow{ }^{\text {a.s. }} 0$.

Proof. The result follows from Lemma 4.1 and Theorem 4.1.

We now proceed to find a condition under which $\liminf _{t \rightarrow \infty} t^{-1 / \alpha}|X(t)|=0$ a.s. As the above theorem indicates this is always true if $\alpha<\min (\theta, \eta)$. We shall prove in a later section that with regard to subordinators with $\gamma>1$, this condition in general cannot be improved. It is, however, true that with regard to certain types of processes $\liminf _{t \rightarrow \infty} t^{-1 / \alpha}|X(t)|=0$ a.s. for all $\alpha>0$. Instead of discussing this situation in all generality we shall restrict ourselves to a particularly simple type of processes i.e. symmetric processes with $\gamma>1$. We require the following definition of recurrence.

Definition 5.1. We shall say that 0 is recurrent with respect to the process $Y=\left\{Y_{n}: n \geqq 1\right\}$ if for every $h>0, P\left[\left|Y_{n}\right|<h\right.$ for an infinity of $\left.n\right]=1$.

THEOREM 5.2. If $\phi(y)$ is real for all $y$ and $\gamma>1$, then $\liminf _{n \rightarrow \infty}|X(n)|=0$ a.s.

Proof. We shall use a theorem due to Chung and Fuchs [3] which states that if for each $n, X_{n}$ be the sum of $n$ independent and identically distributed random variables $Y_{i}, i=1,2, \cdots, n$, then a sufficient condition for 0 to be recurrent value for the process $X^{\prime}=\left\{X_{n}: n \geqq 1\right\}$ is that there exists an $a>0$, such that $\int_{-a}^{a}(1-\phi(u))^{-1} d u=\infty$, where $\phi(u)$ is the characteristic function of $Y_{1}$. In our case, since $\phi(y)$ is real and since $\left|1-e^{-z}\right| \leqq|Z|$ for all complex $Z$ such that $\operatorname{Re} Z \geqq 0$, we obtain $|1-\phi(y)|=1-e^{-\operatorname{Re} \Psi(y)} \leqq \operatorname{Re} \Psi(y)$. Also, since by assumption $\gamma>1$, we can pick by Theorem 4.1 an $\alpha>1$ and an $a>0$, such that $\operatorname{Re} \Psi(y) \leqq|y|^{\alpha}$ if $|y|<a$. Hence,

$$
\int_{-a}^{a}(1-\phi(y))^{-1} d y=\int_{-a}^{a}\left(1-e^{-\operatorname{Re} \Psi(y)}\right)^{-1} d y \geqq \int_{-a}^{a}|y|^{-\alpha} d y=\infty .
$$

The conclusion of the theorem follows from the theorem in [3].

As a consequence of the preceeding theorem we obtain that if $\gamma>1$ and $\phi(y)$ real valued then $\liminf _{t \rightarrow \infty} t^{-1 / \alpha}|X(t)|=0$ a.s. for every $\alpha>0$. We prove in Theorem 6.2 that if $\alpha>\theta$, then $\lim \sup _{t \rightarrow \infty} t^{-1 / \alpha}|X(t)|=\infty$ a.s. These two observa- 
tions lead us to the conclusion that with regard to certain types of processes $\liminf _{t \rightarrow \infty} t^{-1 / \alpha}|X(t)|=0$ a.s. and $\lim \sup _{t \rightarrow \infty} t^{-1 / \alpha}|X(t)|=\infty$ a.s. provided $\alpha>\theta$.

It is interesting to know to what extent the conditions of Theorem 5.2 can be weakened without affecting the conclusion. In $\S \S 6$ and 7 we show that there are processes which satisfy only one of the conditions of Theorem 5.1 and such that the conclusion of the theorem is not applicable. The reader should, however, be reminded before he wants to proceed in this matter, that convergence of the limit inferior to zero has a strong bearing on the recurrence properties of the process and as such demands a more delicate and profound treatment.

6. Divergence to infinity. The main theorem of this section is to show that for a stable process of index $\beta<1, t^{-1 / \alpha}|X(t)| \rightarrow^{\text {a.s. }} \infty$ as $t \rightarrow \infty$, provided $\alpha>\beta /(1-\beta)$. The class of processes which satisfies the property that $t^{-a}|X(t)| \rightarrow^{\text {a.s. }} \infty$ for some suitably chosen $a$, is however, wider than stable processes of index $\beta<1$. In Theorem 6.1 we endeavor to characterize such processes. Let $f(y), G(t, A)$ and $B(t)$ denote the same quantities as in $\S 5$.

Lemma 6.1. If $\theta^{\prime}<1$ and $\alpha>\theta^{\prime} /\left(1-\theta^{\prime}\right)$, then there exists a $c>0$, such that $\operatorname{Sup}_{t \geqq 0} t^{1+c} B(t)<\infty$.

Proof. Now,

$$
\begin{aligned}
B(t) & =\int_{-\infty}^{\infty} e^{-t \Psi\left(x / t^{1 / \alpha}\right)} f(x) d x \\
& =\int_{-\infty}^{\infty} e^{-t \Psi(y)} t^{1 / \alpha} f\left(t^{1 / \alpha} y\right) d y \\
& \leqq \int_{-\infty}^{\infty} t^{1 / \alpha} e^{-t \operatorname{Re} \Psi(y)} f\left(t^{1 / \alpha} y\right) d y .
\end{aligned}
$$

Observe that $\alpha>\theta^{\prime} /\left(1-\theta^{\prime}\right)$ implies that there exists a $\beta>\theta^{\prime}$ an a $c>0$, such that $1+c+1 / \alpha<1 / \beta$. Since $\beta>\theta^{\prime}$, we may assume that there exists a $\delta>0$ such that $\operatorname{Re} \Psi(y) \geqq|y|^{\beta}$, if $|y| \leqq \delta$. Thus,

$$
\begin{aligned}
t^{1+c} B(t) \leqq & \int_{y \leqq \delta} t^{1+c+1 / \alpha} e^{-t|y|^{\beta}} f\left(t^{1 / \alpha} y\right) d y \\
& +\delta^{-(1+c) \alpha} \int_{y \geqq \delta} t^{1+c}|y|^{(1+c) \alpha} t^{1 / \alpha} f\left(t^{1 / \alpha} y\right) d y=I_{1}+I_{2} .
\end{aligned}
$$

Taking $t>1$ and using the facts that $f\left(t^{1 / \alpha} y\right)<K$ and $1+c+1 / \alpha<1 / \beta$ we obtain $I_{1}<K \int_{-\infty}^{\infty} t^{1 / \beta} e^{-\left(t^{1 / \beta}|y|\right)^{\beta}} d y=\int_{-\infty}^{\infty} e^{-|x|^{\beta}} d x=K_{\beta}<\infty$. Likewise $I_{2}<\delta^{-(1+c) \alpha} M_{(1+c) \alpha}$, where $M_{(1+c) \alpha}$ is defined by (5.1). Thus

$$
t^{1+c} B(t)<K_{\beta}+\delta^{-(1+c) \alpha} M_{(1+c) \alpha}
$$

for all $t>1$. Also, since for $t \leqq 1, t^{1+c} B(t) \leqq 1$, the lemma is proved. 
THEOREM 6.1. If $\theta^{\prime}<1$ and $\alpha>\theta^{\prime} / 1-\theta^{\prime}$, then $t^{-1 / \alpha}|X(t)| \rightarrow^{\text {a.s. }} \infty$ as $t \rightarrow \infty$.

Proof. Since $\alpha>\theta^{\prime} /\left(1-\theta^{\prime}\right)$, we obtain by Lemma 6.1 :

$$
\begin{aligned}
K t^{-(1+c)}>B(t) & \geqq \int_{x \leqq \eta} e^{-x^{2}} G(t, d x) \geqq e^{-\eta^{2}} \int_{x \leqq \eta} G(t, d x) \\
& =e^{-\eta^{2}} P\left[t^{-1 / \alpha}|X(t)| \leqq \eta\right] \text { for every } \eta>0 .
\end{aligned}
$$

Thus,

$$
P\left[t^{-1 / \alpha}|X(t)| \leqq \eta\right]<C_{\eta} t^{-(1+c)}
$$

Hence,

$$
\int_{1}^{\infty} P\left[t^{-1 / \alpha}|X(t)| \leqq \eta\right] d t<\infty
$$

We shall show that (6.2) implies that $\liminf _{t \rightarrow \infty} t^{-1 / \alpha}|X(t)|=\infty$ a.s. By the zeroone law $\liminf _{t \rightarrow \infty} t^{-1 / \alpha}|X(t)|=R$ a.s. Hence, if $R<\infty$, there exists an $h>0$ such that $R \in(-h, h)$ and such that

$$
P\left[\omega|| t^{-1 / \alpha} X(t, \omega) \mid \leqq h \text { for some } t>N, N=1,2, \cdots\right]=1 .
$$

Define

$$
T_{1}(\omega)=\inf \left\{t \geqq 1\left|t^{-1 / \alpha}\right| X(t, \omega) \mid>3 h\right\} .
$$

Since (6.1) is true for all $\eta>0$, it follows from the Borel-Cantelli Lemma that the sequence of random variables $n^{-1 / \alpha} X(n), n=1,2, \cdots$ diverges to infinity with probability one as $n \rightarrow \infty$. Thus, $T_{1}(\omega)<\infty$ with probability one. Having defined $T_{1}(\omega)$ we define

$$
S_{1}(\omega)=\inf \left\{t \geqq T_{1}(\omega)\left|t^{-1 / \alpha}\right| X(t, \omega) \mid \leqq h\right\} .
$$

By assumption (6.3), $S_{1}(\omega)<\infty$ with probability one. We define $T_{n}(\omega), S_{n}(\omega)$ as follows:

$$
\begin{gathered}
T_{2}(\omega)=\inf \left\{t \geqq S_{1}(\omega)\left|t^{-1 / \alpha}\right| X(t, \omega) \mid>3 h\right\}, \\
\vdots \\
S_{n}(\omega)=\inf \left\{t \geqq T_{n}(\omega)\left|t^{-1 / \alpha}\right| X(t, \omega) \mid \leqq h\right\}, \\
T_{n+1}(\omega)=\inf \left\{t \geqq S_{n}(\omega)\left|t^{-1 / \alpha}\right| X(t, \omega) \mid>3 h\right\} .
\end{gathered}
$$

Observe that each of $T_{n}(\omega)$ and $S_{n}(\omega)$ for $n=1,2, \cdots$ is finite with probability one. Let $T_{h}(\omega)=\mu\left[t \in[1, \infty)\left|t^{-1 / \alpha}\right| X(t, \omega) \mid \leqq 3 h\right]$, where $\mu$ represents the Lebesgue measure on $[1, \infty)$. Obviously, $T_{h}(\omega) \geqq \sum_{n=2}^{\infty}\left[T_{n}(\omega)-S_{n-1}(\omega)\right]$. Hence $\mathscr{E} T_{h}(\omega) \geqq \sum_{n=2}^{\infty} \mathscr{E}\left[T_{n}(\omega)-S_{n-1}(\omega)\right]$.

For any linear Borel set $T$ of $[1, \infty)$ define

$$
F_{n}(T)=P\left[\omega \mid S_{n}(\omega) \in T\right] .
$$


Fix a $t \geqq 1$. For any $s>t$, denote by $A_{t}^{s}$ the event $\left|t^{-1 / \alpha} X(t)-s^{-1 / \alpha} X(s)\right|>2 h$. Thus,

$$
\begin{aligned}
A_{t}^{s} & =\left\{\left|t^{-1 / \alpha} X(t)-s^{-1 / \alpha} X(s)\right|>2 h\right\} \\
& =\left\{t^{-1 / \alpha}\left|\left(t s^{-1}\right)^{1 / \alpha} X(s)-\left(t s^{-1}\right)^{1 / \alpha} X(t)+\left(t s^{-1}\right)^{1 / \alpha} X(t)-X(t)\right|>2 h\right\} .
\end{aligned}
$$

Since $s>t \geqq 1$ we have

Thus,

$$
A_{t}^{s} \subset\{|X(s)-X(t)|>h\} \cup\left\{|X(t)|>h t^{1 / \alpha}\right\} .
$$

$$
\begin{aligned}
\left\{\begin{array}{l}
\text { L.U.B. } \\
t \leqq s \leqq t+\tau
\end{array}\left|t^{-1 / \alpha} X(t)-s^{-1 / \alpha} X(s)\right|>2 h\right\} \\
\subset\{\underset{t \leqq s \leqq t+\tau}{\operatorname{L.U.B.} \mid}|X(s)-X(t)|>h\} \cup\left\{|X(t)|>h t^{1 / \alpha}\right\} .
\end{aligned}
$$

For the sake of brevity we shall denote the event on the left side of (6.4) by $B^{t}$, the first event of the right side by $B_{1}^{t}$ and the second event of the right side by $B_{2}^{t}$. Let $\varepsilon$ be such that $0<\varepsilon<1 / 2$. Since the process has stationary independent increments it is possible to find a $\tau>0$, such that

$$
P[|X(t)| \leqq h / 2]>1-\varepsilon \text { holds for all } t \leqq \tau .
$$

We may assume that $\tau$ that appears in (6.4) is chosen that way. Comparing (6.5) with (2.3) we obtain

$$
P[\underset{t \leqq s \leqq t+\tau}{\operatorname{L.U.B}}|X(s)-X(t)|>h] \leqq \frac{\varepsilon}{1-\varepsilon}=\delta<1
$$

by (2.4). Thus,

$$
\begin{aligned}
P\left[T_{n}-S_{n-1}<\tau\right] & \leqq \int_{1}^{\infty} P\left[B^{t} / S_{n-1}=t\right] d F_{n-1}(t) \\
& \leqq \int_{1}^{\infty} P\left[B_{1}^{t} / S_{n-1}=t\right] d F_{n-1}(t)+\int_{1}^{\infty} P\left[B_{2}^{t} / S_{n-1}=t\right] d F_{n-1}(t) \\
& =I_{1}+I_{2} .
\end{aligned}
$$

But, since the process has stationary independent increments

$$
I_{1}=\int_{1}^{\infty} P\left(B_{1}^{t}\right) d F_{n-1}(t) \leqq \delta<1,
$$

by (6.6). Also, the integrand in $I_{2}$ is equal to zero. Thus, $P\left[T_{n}-S_{n-1} \geqq \tau\right]$ $\geqq 1-\delta>0$. This implies, $\mathscr{E}\left(T_{n}-S_{n-1}\right) \geqq(1-\delta) \tau$. Thus $\mathscr{E} T_{h}=\infty$. Whereas, $\mathscr{E} T_{h}=\int_{\Omega} P(d \omega) \int_{1}^{\infty} I\left[t^{-1 / \alpha}|X(t, \omega)| \leqq 3 h\right] d t$, where $I$ denotes the indicator function of $\left\{(t, \omega)\left|t^{-1 / \alpha}\right| X(t, \omega) \mid \leqq 3 h\right\}$. Thus 


$$
\begin{aligned}
\mathscr{E} T_{h} & =\int_{1}^{\infty} d t \int_{\Omega} I\left\{t^{-1 / \alpha}|X(t, \omega)| \leqq 3 h\right\} P(d \omega) \\
& =\int_{1}^{\infty} P\left[t^{-1 / \alpha}|X(t, \omega)| \leqq 3 h\right] d t<\infty,
\end{aligned}
$$

by (6.2). Thus, we arrive at a contradiction. Hence, $\liminf _{t \rightarrow \infty} t^{-1 / \alpha}|X(t)|=\infty$ a.s. and the proof is completed.

For a stable process of index $\beta$, we have $\gamma=\beta=\theta=\theta^{\prime}$. Therefore if $\beta<1$ and $\alpha>\beta /(1-\beta)$ then we obtain by Theorem 6.1 that $t^{-1 / \alpha}|X(t)| \rightarrow^{\text {a.s. }} \infty$, as claimed at the beginning of this section. We should remark at this stage that an interesting problem for carrying out further research is to investigate the behavior of the sample functions of the process

$$
Y_{1 / \alpha}=\left\{t^{-1 / \alpha} X(t): t \geqq 0\right\}, \text { where } X=\{X(t): t \geqq 0\}
$$

is a process with stationary independent increments with $\theta^{\prime}<1$ and $\alpha$ is such that $\theta<\alpha<\theta^{\prime} /\left(1-\theta^{\prime}\right)$.

The following theorems give us the conditions under which

$$
\limsup _{t \rightarrow \infty} t^{-1 / \alpha}|X(t)|=\infty \text { a.s. }
$$

THEOREM 6.2. If $\alpha>\theta$, then $\lim \sup _{t \rightarrow \infty} t^{-1 / \alpha}|X(t)|=\infty$ a.s.

Proof. The proof is similar to the proof of Theorem 3.3 of [1].

Suppose next we have a process with $\gamma>\eta$ and $\eta=1$. By Theorem 5.1 $t^{-1 / \alpha} X(t) \rightarrow{ }^{\text {a.s. }} 0$ for all $\alpha<1$ and by the preceding theorem $\lim \sup _{t \rightarrow \infty} t^{-1 / \alpha}|X(t)|$ $=\infty$ a.s. for all $\alpha$ such that $\alpha>\min (2, \gamma)$. What can one say about $t^{-1 / \alpha}|X(t)|$ for all $\alpha$ such that $1<\alpha<\min (2, \gamma)$ ? In the following theorem we endeavor to give a partial answer to this question in a more general form than above.

THEOREM 6.3. For every $\alpha>\eta^{\prime}$, $\quad \lim \sup _{t \rightarrow \infty} t^{-1 / \alpha} X(t)=\infty$ a.s.

Proof. The only case that we need to consider is when $1 \leqq \eta^{\prime}<\theta$.

The reader will not have any difficulty in verifying for himself that the proofs of all other different cases follow from the preceding theorem. So, let $1 \leqq \eta^{\prime}<\theta$. It suffices to prove the theorem for an $\alpha$ such that $\eta^{\prime}<\alpha<\theta$, otherwise chosen arbitrarily. Take an $\alpha^{\prime}$ such that $\eta^{\prime}<\alpha^{\prime}<\alpha$. If possible let limsup $\sup _{t \rightarrow \infty} t^{-1 / \alpha} X(t)$ $=R<\infty$ a.s. Then $t^{-1 / \alpha^{\prime}} X(t) \rightarrow^{\text {a.s. }} 0$. Consider the corresponding characteristic function $\phi_{t}(y)$ of $t^{-1 / \alpha^{\prime}} X(t)$ for positive values of $y$ only.

$$
\begin{aligned}
\phi_{t}(y)= & \left.e^{-t \Psi\left(y / t^{1 / \alpha^{\prime}}\right)}=e^{-t \operatorname{Re} \Psi\left(y / t^{1 / \alpha^{\prime}}\right)} \times e^{-i t \operatorname{Im} \Psi\left(y / t^{1 / \alpha^{\prime}}\right.}\right) \\
= & \exp \left[-t \operatorname{Re} \Psi\left(y / t^{1 / \alpha^{\prime}}\right)\left(y / t^{1 / \alpha^{\prime}}\right)^{-\alpha^{\prime}}\left(y / t^{1 / \alpha^{\prime}}\right)^{\alpha^{\prime}}\right] \\
& \times \exp \left[-i t \operatorname{Im} \Psi\left(y / t^{1 / \alpha^{\prime}}\right)\left(y / t^{1 / \alpha^{\prime}}\right)^{\alpha^{\prime}}\left(y / t^{1 / \alpha^{\prime}}\right)^{-\alpha^{\prime}}\right] .
\end{aligned}
$$


We investigate separately each of the factors that appear on the right side of (6.7).

$$
\begin{aligned}
& \exp \left[-t \operatorname{Re} \Psi\left(y / t^{1 / \alpha^{\prime}}\right)\left(y / t^{1 / \alpha^{\prime}}\right)^{-\alpha^{\prime}}\left(y_{/} \iota^{1 / \alpha^{\prime}}\right)^{\alpha^{\prime}}\right] \\
& =\exp \left[-\operatorname{Re} \Psi\left(y / t^{1 / \alpha^{\prime}}\right)\left(y / t^{1 / \alpha^{\prime}}\right)^{-\alpha^{\prime}} y^{\alpha^{\prime}}\right]
\end{aligned}
$$

Since $\alpha^{\prime}<\theta$, for every fixed $y$ the right side of the above expression approaches one as $t \rightarrow \infty$.

After suitable reduction the second factor of (6.7) can be written as $\exp \left[-i \operatorname{Im} \Psi\left(y / t^{1 / \alpha^{\prime}}\right)\left(y / t^{1 / \alpha^{\prime}}\right)^{-\alpha^{\prime}} y^{\alpha^{\prime}}\right]$. Since $\operatorname{Im} \Psi(y)$ is a continuous function of $y$ and $\alpha^{\prime}>\eta^{\prime}$, for every fixed $y>0, \operatorname{Im} \Psi\left(y / t^{1 / \alpha^{\prime}}\right)\left(y / t^{1 / \alpha^{\prime}}\right)^{-\alpha^{\prime}} y^{\alpha^{\prime}} \rightarrow \pm \infty$ as $t \rightarrow \infty$ and therefore the second factor of (6.7) does not approach any limit whatsoever. This observation together with the fact that $e^{-t R^{e} \Psi\left(y / t^{1 / \alpha^{\prime}}\right)} \rightarrow 1$ as $t \rightarrow \infty$ leads us to the conclusion that $\phi_{t}(y)$ does not converge to any limit whatsoever. This in turn implies that $t^{-1 / \alpha} X(t)$ does not converge to zero with probability one. This, however, is a contradiction which completes the proof.

Most of the processes that one encounters in practice satisfy the relation $\theta=\theta^{\prime}$. That this is not true in general, will be shown by means of an example in a later section. The following theorem shows that if in Theorem 6.2 we replace the condition $\alpha>\theta$ by $\alpha>\theta^{\prime}$, we get more interesting results.

THEOREM 6.4. If $\alpha>\theta^{\prime}$, then

(1) $t^{-1 / \alpha}|X(t)| \rightarrow \infty$ in probability as $t \rightarrow \infty$.

(2) $\left(2^{n}\right)^{-1 / \alpha}\left|X\left(2^{n}\right)\right| \rightarrow^{\text {a.s. }} \infty$ as $n \rightarrow \infty$.

Proof. By taking a $\beta$ such that $\theta^{\prime}<\beta<\alpha$ and following the same line of argument as in Lemma 6.1, one can prove that if $c$ be a quantity such that $0<c<1 / \beta-1 / \alpha$, then Sup $t^{c} B(t)<K<\infty$; whence it follows that $P\left[t^{-1 / \alpha}|X(t)| \leqq M\right]<K_{m} t^{-c}$. Conclusions of Theorem 6.4 become immediate.

7. Subordinators. In this section we shall assume that the support of the Lévy measure is contained in the half line $x>0$ and that $\int_{0}^{1} x d v(x)<\infty$. In this case the exponent $\Psi(y)$ can be written in the form

$$
\Psi(y)=i c y+\sigma^{2} y^{2}+\int_{0}^{\infty}\left(1-e^{-i x y}\right) v(d x) .
$$

We shall restrict ourselves to those cases where $c=0$ and $\sigma^{2}=0$. Such a process is called a subordinator. It is known that a subordinator has the additional property that the sample functions are nondecreasing with probability one. It can be proved that if $X$ is a subordinator, the Laplace transform $L_{t}(u)$ of $X_{t}(\omega)$ exists for $u>0$ and is given by

$$
L_{t}(u)=\mathscr{E} e^{-u X(t)}=e^{-t g(u)} \quad(u \geqq 0),
$$

where $g$ is necessarily of the form 


$$
g(u)=\int_{0}^{\infty}\left(1-e^{-r u}\right) d v(r),
$$

where $v$, as before, represents the Lévy measure.

The exponent $g$ is often referred to as the subordinator exponent. For an elaborate discussion of subordinators see [2, $\S 4.1$ and 4.3]. In order to avoid repetition we shall assume that $v \not \equiv 0$. According to the notations for this section, the indices $\delta$ and $\delta^{\prime}$ defined in $\S 3$ assume the forms

$$
\begin{aligned}
\delta & =\sup \left[\alpha: u^{-\alpha} g(u) \rightarrow 0 \text { as } u \rightarrow 0\right], \\
\delta^{\prime} & =\inf \left[\alpha: u^{-x} g(u) \rightarrow \infty \text { as } u \rightarrow 0\right] .
\end{aligned}
$$

Obviously $\delta \leqq \delta^{\prime}$. We first state the following

Lemma 7.1. The index $\delta^{\prime}$ satisfies the inequality $\delta^{\prime} \leqq 1$.

Proof. Obvious.

THEOREM 7.1. The indices $\delta$ and $\gamma$ satisfy the relation $\delta=\min (1, \gamma)$.

Proof. Except for trivial modifications the proof can be written in precisely the same fashion as the proof of Theorem 4.1.

Define

$$
C(t)=\int_{0}^{\infty} \frac{1}{x+1} G(t, d x)
$$

where $G(t, A)$ is the distribution of the random variable $t^{-1 / \alpha} X(t)$. We have

$$
\begin{aligned}
C(t) & =\int_{0}^{\infty} \frac{1}{x+1} G(t, d x)=\int_{0}^{\infty} G(t, d x) \int_{0}^{\infty} e^{-x u} e^{-u} d u \\
& =\int_{0}^{\infty} e^{-t g\left(u / t^{1 / \alpha}\right.} e^{-u} d u .
\end{aligned}
$$

Lemma 7.2. For any $\alpha>\delta^{\prime}$ there exists a $c>0$, such that $\operatorname{Sup}_{t \geq 0} t^{c} C(t)<\infty$.

Proof. Take a $\beta$ such that $\delta^{\prime}<\beta<\alpha$ and then choose $c>0$, to be any quantity such that $c<1 / \beta-1 / \alpha$. Next let $a$ be such that $g(u)>u^{\beta}$ provided $0<u<a$. The reader can verify that $t^{c} C(t) \leqq \int_{0}^{\infty} e^{-x^{\beta}} d x+a^{-c \alpha} \Gamma(c \alpha+1)$ for all $t>1$. Also, since for $t \leqq 1, t^{c} C(t) \leqq 1$, the proof is completed.

THEOREM 7.2. Let $X$ be a subordinator. Then

(1) If $\alpha>\delta^{\prime}, t^{-1 / \alpha} X(t) \rightarrow^{\text {a.s. }} \infty$ as $t \rightarrow \infty$;

(2) If $\alpha<\delta^{\prime}, \liminf _{t \rightarrow \infty} t^{-1 / \alpha} X(t)=0$ a.s.

Proof. In order to prove (1) we observe that by Lemma 7.2 we obtain for every $M>0$, 


$$
K t^{-c}>C(t) \geqq \int_{0}^{M} \frac{1}{x+1} G(t, d x) \geqq \frac{1}{M+1} P\left[t^{-1 / \alpha} X(t) \leqq M\right]
$$

Thus,

$$
P\left[t^{-1 / \alpha} X(t)<M\right]<K_{M} t^{-c},
$$

for every $M>0$.

It follows from (7.3) that the sequence of random variables $2^{-n / \alpha} X\left(2^{n}\right)$ diverges to infinity with probability one, i.e., there exists a set $\Omega_{0}$ with $P\left(\Omega_{0}\right)=1$ such that if $\omega \in \Omega_{0}, 2^{-n / \alpha} X\left(2^{n}, \omega\right) \rightarrow \infty$ as $n \rightarrow \infty$. Using the fact that $X$ is a subordinator we may assume without any loss of generality, that if $\omega \in \Omega_{0}, X(t, \omega) \geqq X(s, \omega)$ whenever $t>s$. Take $t$ such that $2^{n}<t \leqq 2^{n+1}$. Then if $\omega \in \Omega_{0}$ we have

$$
t^{-1 / \alpha} X(t, \omega) \geqq 2^{-(n+1) / \alpha} X\left(2^{n}, \omega\right)=2^{-1 / \alpha} 2^{-n / \alpha} X\left(2^{n}, \omega\right) .
$$

Thus, if $\omega \in \Omega_{0}, t^{-1 / \alpha} X(t, \omega) \rightarrow \infty$ as $t \rightarrow \infty$.

We now prove (2). It follows from the definition of $\delta^{\prime}$ that there exists a sequence $\left\{u_{n}\right\}$ with $u_{n} \rightarrow 0$ such that $g\left(u_{n}\right) u_{n}^{-\alpha} \rightarrow 0$. Setting $t_{n}=u_{n}^{-\alpha}$ we observe that $t_{n} \rightarrow \infty$ as $n \rightarrow \infty$ and that

$$
\mathscr{E} \exp \left[-t_{n}^{-1 / \alpha} X\left(t_{n}\right)\right]=\exp \left[-t_{n} g\left(t_{n}^{-1 / \alpha}\right)\right] \rightarrow 1 .
$$

Thus, $t_{n}^{-1 / \alpha} X\left(t_{n}\right) \rightarrow 0$ in probability as $n \rightarrow \infty$ and there exists a subsequence $\left\{t_{n k}\right\}$ of $\left\{t_{n}\right\}$ such that $t_{n_{k}}^{-1 / \alpha} X\left(t_{n_{k}}\right) \rightarrow^{\text {a.s. }} 0$ as $k \rightarrow \infty$.

THEOREM 7.3. Let $X$ be a subordinator. Then, $\delta \leqq \theta$ and $\delta^{\prime} \leqq \theta^{\prime}$.

Proof. The first part of the proof follows from the fact, $\delta=\min (1, \gamma)$ $\leqq \min (2, \gamma)=\theta$. In order to prove the second part, suppose $\theta^{\prime}<\delta^{\prime}$ holds. Take an $\alpha$ such that $\theta^{\prime}<\alpha<\delta^{\prime}$. Since $\alpha$ is greater than $\theta^{\prime}$, we obtain by Theorem 6.4 that $2^{-n / \alpha} X\left(2^{n}\right) \rightarrow{ }^{\text {a.s. }} \infty$ as $n \rightarrow \infty$. But this condition implies (see the proof of Theorem 7.2) that $t^{-1 / \alpha} X(t) \rightarrow$ a.s. $\infty$. But since $\alpha<\delta^{\prime}$, this contradicts Theorem 7.2. Thus, $\delta^{\prime} \leqq \theta^{\prime \prime}$.

We want to conclude this paper by making a remark that one can construct a subordinator with $\theta=\gamma=\delta<\delta^{\prime}$ (and hence a process with $\theta<\theta^{\prime}$ ) using essentially the same method as used in $\$ 7$ of [1].

8. Acknowledgements. The author wishes to express his gratitude to Professor R. M. Blumenthal for suggesting this topic and for his advice and comments while this paper was being written. The author also wants to thank Professor R. Pyke for his valuable suggestions in this connection, and the N.S.F. (Grant G 14248) for assisting him financially during the later part of his work. 


\section{BIBLIOGRAPHY}

1. R. M. Blumenthal and R. K. Getoor, Sample functions of stochastic processes with stationary independent increments, J. Math. Mech. 10 (1961), 493-516.

2. S. Bochner, Harmonic analysis and the theory of probability, Univ. of California Press, Berkeley, Calif. 1955.

3. K. L. Chung and W. H. J. Fuchs, On the distribution of sums of random variables, Mem. Amer. Math. Soc. No. 6 (1951), pp. 1-12.

4. A. Khintchine, Zwei Satze über stochastische Prozesse mit stabilen Verteilungen, Mat. Sb. (N. S.) 3 (1938), 584.

5. - Sur la croissance locale des processes stochastique homogènes à accroissements independants, Izv. Akad. Nauk SSSR Ser. Mat. (1939), 487-508.

UNIVERSITY OF IDAHO,

Moscow, IDAHO

\section{ERRATA TO VOLUME 107}

A. H. Stone, Kernel constructions and Borel sets, pp. 58-70.

The paper referred to (these Transactions, Vol. 107, pp. 58-70) should be amended as follows.

(1) In the corollary to Theorem 9 (p. 68), $\xi$ should be required to be $>0$ in the additive case also.

(2) The list of references should have included: E. Michael, Local properties of topological spaces, Duke Math. J. 21 (1954), 163-172. Lemma 4 of Kernel constructions is an easy consequence of Michael's Theorem 3.6.

(3) Lemma 3 and its corollary are true without any hypotheses on $Y$, and are proved in this form in reference [5, p. 49]. Theorem 8 is also true without the hypotheses on $Y$, as may be shown by an argument similar to that proving Theorem 9. 